\title{
A CONVERGENCE RESULT FOR SQUARE ROOTS OF THE POISSON KERNEL IN THE BIDISK
}

\author{
JAN-OLAV RÖNNING
}

\begin{abstract}
Let $P(z, \beta)$ be the Poisson kernel in the unit disk U. For $f \in L^{p}\left((\partial \mathrm{U})^{2}\right)$ and $\left(z_{1}, z_{2}\right) \in \mathrm{U}^{2}$, let $P_{\lambda_{1}, \lambda_{2}} f$ be the integral of $f$ against the kernel $P\left(z_{1}, \varphi_{1}\right)^{\frac{1}{2}+\lambda_{1}} P\left(z_{2}, \varphi_{2}\right)^{\frac{1}{2}+\lambda_{2}}$ and let $\mathscr{P}_{\lambda_{1}, \lambda_{2}} f$ be the normalization $P_{\lambda_{1}, \lambda_{2}} f / P_{\lambda_{1}, \lambda_{2}} 1$.

In a previous paper, we proved that $P_{\lambda_{1}, 0} f\left(z_{1}, z_{2}\right)$ converges to $f\left(\beta_{1}, \beta_{2}\right)$ in a region $A \times B$, where $A$ is a nontangential region and $B$ is a slightly larger convex region, for a.a. $\left(\beta_{1}, \beta_{2}\right) \in(\partial \mathrm{U})^{2}$. A natural question to ask next is if the convergence regions for $P_{0,0} f$ are products of these larger convex regions if $f \in L^{p}\left((\partial \mathrm{U})^{2}\right)$. This paper will show that this is the case if we consider restricted convergence, that is, if the variables $z_{1}$ and $z_{2}$ tend to the boundary $(\partial \mathrm{U})^{2}$ with approximately the same speed.
\end{abstract}

\section{Introduction}

Let

$$
P(z, \beta)=\frac{1}{2 \pi} \frac{1-|z|^{2}}{\left|z-e^{i \beta}\right|^{2}}, z=x+i y \in \mathrm{U}, e^{i \beta} \in \mathrm{T}=\partial \mathrm{U}
$$

be the standard Poisson kernel in the unit disk U. A well-known Fatou type result states that the Poisson integral

$$
P f(x)=\int_{\mathrm{T}} P(z, \varphi) f(\varphi) d \varphi
$$

of a function $f \in L^{p}(\mathrm{~T})$ converges to $f(\beta)$ as $z$ tends to $e^{i \beta}$ nontangentially, for a.a. $e^{i \beta} \in \mathrm{T}$. Here we let $f(\beta)$ stand for $f\left(e^{i \beta}\right)$ when $f$ is defined on $\mathrm{T}$.

Let

$$
P_{\lambda} f(z)=\int_{\mathrm{T}} P(z, \varphi)^{\lambda+1 / 2} f(\varphi) d \varphi, \quad \lambda \geq 0,
$$

where $f \in L^{p}(\mathrm{~T}), 1 \leq p \leq \infty$.

Received August 5, 1996. 
We know that $P_{\lambda} f(z)$ satisfies the equation $L_{z} u=\left(\lambda^{2}-1 / 4\right) u$, where

$$
L_{z}=\frac{1}{4}\left(1-|z|^{2}\right)^{2}\left(\frac{\partial^{2}}{\partial x^{2}}+\frac{\partial^{2}}{\partial y^{2}}\right)
$$

is the hyperbolic Laplacian.

Because $P_{\lambda} 1(z)$ does not converge to 1 as $z$ tends to $e^{i \beta}$, we need to consider the normalization

$$
\mathscr{P}_{\lambda} f(z)=\frac{P_{\lambda} f(z)}{P_{\lambda} 1(z)}
$$

to obtain a.e. convergence to $f\left(e^{i \beta}\right)$ if $f \in L^{p}(\mathrm{~T}), 1 \leq p \leq \infty$. If $\lambda>0$, we know that $\mathscr{P}_{\lambda} f(z)$ converges to $f\left(e^{i \beta}\right)$ as $z$ tends to $e^{i \beta}$ nontangentially.

If $\lambda=0$, we have a.e. convergence of $\mathscr{P}_{0} f(z)$ to $f\left(e^{i \beta}\right)$ in the $L^{p}$ weakly tangential regions

$$
\left\{z \in \mathrm{U} ;|\arg (z)-\beta| \leq A(1-|z|)\left(\log \frac{1}{1-|z|}\right)^{p}\right\},
$$

for $f \in L^{p}(\mathbf{T}), 1 \leq p<\infty$. Here $A$ is a fixed constant. This was proved in [1]. For $p=1$, this result is the same as the result in [2].

In [1] we also investigated the convergence of the operator

$$
\mathscr{P}_{\lambda_{1}, 0} f\left(z_{1}, z_{2}\right)=\frac{P_{\lambda_{1}, 0} f\left(z_{1}, z_{2}\right)}{P_{\lambda_{!}, 0} 1\left(z_{1}, z_{2}\right)}
$$

in the bidisk. The operators $P_{\lambda_{1}, \lambda_{2}} f\left(z_{1}, z_{2}\right)$ are defined by

$$
P_{\lambda_{1}, \lambda_{2}} f\left(z_{1}, z_{2}\right)=\int_{\mathrm{T}^{2}} P\left(z_{1}, \varphi_{1}\right)^{\lambda_{1}+1 / 2} P\left(z_{2}, \varphi_{2}\right)^{\lambda_{2}+1 / 2} f\left(\varphi_{1}, \varphi_{2}\right) d \varphi_{1} d \varphi_{2}
$$

for $f \in L^{p}\left(\mathrm{~T}^{2}\right), 1 \leq p \leq \infty$.

We proved that $\mathscr{P}_{\lambda_{1}, 0} f\left(z_{1}, z_{2}\right)$ converges restrictedly a.e. to $f\left(e^{i \beta_{1}}, e^{i \beta_{2}}\right)$, if $\lambda_{1}>0, f \in L^{1}\left(\mathrm{~T}^{2}\right)$ and $\left(z_{1}, z_{2}\right)$ tends to $\left(e^{i \beta_{1}}, e^{i \beta_{2}}\right)$ in a product region $A \times B$ of $\mathrm{U}^{2}$. Here $A$ is a nontangential region and $B$ is a weakly tangential region, and restricted convergence means that $z_{1}$ and $z_{2}$ tend to the boundary equally fast. This is, however, not the best result we can prove if we allow $\lambda_{1}$ to be 0 .

In this paper we will extend the bidisk result in [1] accordingly. We will assume that $f \in L^{p}\left(\mathrm{~T}^{2}\right), 1<p<\infty$, and show that $\mathscr{P}_{0,0} f\left(z_{1}, z_{2}\right)$ converges restrictedly a.e. to $f\left(e^{i \beta_{1}}, e^{i \beta_{2}}\right)$ as $\left(z_{1}, z_{2}\right)$ tends to $\left(e^{i \beta_{1}}, e^{i \beta_{2}}\right)$ in a region $A \times B$, where both $A$ and $B$ are $L^{p}$ weakly tangential regions. For $f \in L^{1}\left(\mathrm{~T}^{2}\right)$ this was proved by Sjögren in [3].

We will prove this result by establishing the usual weak type $(p, p)$ esti- 
mate for the maximal function $\sup _{\left(z_{1}, z_{2}\right) \in A \times B} \mathscr{P}_{0,0}|f|\left(z_{1}, z_{2}\right)$. In order to do this, we use Lemma 1 in [1].

With a slight abuse of notation, we will identify $e^{i \beta} \in \mathrm{U}$ and $\left(e^{i \beta_{1}}, e^{i \beta_{2}}\right) \in \mathrm{U}^{2}$ with $\beta \in \mathrm{R}$ and $\left(\beta_{1}, \beta_{2}\right) \in \mathbf{R}^{2}, 0 \leq \beta, \beta_{1}, \beta_{2} \leq 2 \pi$, respectively. We let $C$ denote various constants, and the notation $f \sim g$ means that there exist two constants $0<k<K<\infty$, not necessarily the same from time to time, such that $k<f / g<K$. For further references, see the list in [1].

\section{The convergence result}

Let $\left(z_{1}, z_{2}\right)=\left(r_{1} e^{i \theta_{1}}, r_{2} e^{i \theta_{2}}\right) \in \mathrm{U}^{2}$, and define $A_{\beta_{1}, \beta_{2}}^{p}$ to be the sets

$$
\begin{aligned}
& A_{\beta_{1}, \beta_{2}}^{p}=\left\{\left(z_{1}, z_{2}\right) ; 1-r_{1} \sim 1-r_{2}, 1 / 2<r_{j}<1,\right. \\
& \left.\left|\beta_{j}-\theta_{j}\right| \leq\left(1-r_{j}\right)\left(\log \frac{1}{1-r_{j}}\right)^{p}, j=1,2\right\} .
\end{aligned}
$$

We are now going to prove the following theorem:

Theorem 1. Let $f \in L^{p}\left(\mathrm{~T}^{2}\right), 1<p<\infty$. Then $\mathscr{P}_{0,0} f\left(z_{1}, z_{2}\right)$ converges to $f\left(\beta_{1}, \beta_{2}\right)$ as $\left(z_{1}, z_{2}\right)$ tends to $\left(\beta_{1}, \beta_{2}\right)$ in $A_{\beta_{1}, \beta_{2}}^{p}$, for a.a. $\left(\beta_{1}, \beta_{2}\right) \in \mathrm{T}^{2}$.

As usual, this follows directly from the following maximal function estimate.

THeOREM 2. Let

$$
M_{A^{p}} f\left(\beta_{1}, \beta_{2}\right)=\sup _{\left(z_{1}, z_{2}\right) \in A_{\beta_{1}, \beta_{2}}^{p}} \mathscr{P}_{0,0}|f|\left(z_{1}, z_{2}\right) .
$$

Then $M_{A^{p}}$ is of weak type $(p, p), 1<p<\infty$.

In the proof of Theorem 2, we make use of a lemma from [1] which we state here for easy reference.

Lemma 1. Assume that the operators $T_{k}, k=1,2, \ldots$ are defined in $\mathrm{T}^{n}$ by

$$
T_{k} f(x)=\sup _{s \in I_{k}} K_{s} *|f|(x),
$$

where the $K_{s}$ are nonnegative and integrable in $\mathrm{T}^{n}$, and $K_{s}$ and the index sets $I_{k}$ are such that $T_{k} f$ is measurable for any measurable function $f$. Let, for each $i=1, \ldots, n$, a decreasing sequence $\left\{\gamma_{k i}\right\}_{k=1}^{\infty}$ be given, and assume that the $T_{k}$ are of weak type $(p, p)$ with constant at most $C_{0}$ for some $p, 1 \leq p<\infty$, where the constant $C_{0}$ depends on $p$ but not on $k$. Also assume that 
(1) $\operatorname{supp} K_{s} \subset\left\{x=\left(x_{1}, \ldots, x_{n}\right) \in \mathrm{T}^{n} ;\left|x_{i}\right| \leq \gamma_{k i}, i=1, \ldots, n\right\}, s \in I_{k}$,

and, denoting

$$
K_{s}^{*}(x)=\sup \left\{K_{s}(x+y) ;\left|y_{i}\right| \leq \gamma_{k+N, i}, i=1, \ldots, n\right\}
$$

for $s \in I_{k}$ and some natural number $N$,

$$
\int K_{s}^{*}(x) d x \leq C_{0}, \quad s \in \cup_{k} I_{k}
$$

Then the operator

$$
T f(x)=\sup _{k} T_{k} f(x)
$$

Assume that the operators $T_{k}, k=1,2, \ldots$ are defined in $\mathrm{T}^{n}$ by

$$
T_{k} f(x)=\sup _{s \in I_{k}} K_{s} *|f|(x),
$$

where the $K_{s}$ are nonnegative and integrable in $\mathrm{T}^{n}$, and $K_{s}$ and the index sets $I_{k}$ are such that $T_{k} f$ is measurable for any measurable function $f$. Let, for each $i=1, \ldots, n$, a decreasing sequence $\left\{\gamma_{k i}\right\}_{k=1}^{\infty}$ be given, and assume that the $T_{k}$ are of weak type $(p, p)$ with constant at most $C_{0}$ for some $p, 1 \leq p<\infty$. Also assume that

(3) $\operatorname{supp} K_{s} \subset\left\{x=\left(x_{1}, \ldots, x_{n}\right) \in \mathrm{T}^{n} ;\left|x_{i}\right| \leq \gamma_{k i}, i=1, \ldots, n\right\}, s \in I_{k}$,

and, denoting

$$
K_{s}^{*}(x)=\sup \left\{K_{s}(x+y) ;\left|y_{i}\right| \leq \gamma_{k+N, i}, i=1, \ldots, n\right\}
$$

for $s \in I_{k}$ and some natural number $N$,

$$
\int K_{s}^{*}(x) d x \leq C_{0}, \quad s \in \cup_{k} I_{k}
$$

Then the operator

$$
T f(x)=\sup _{k} T_{k} f(x)
$$

is of weak type $(p, p)$ with constant depending only on $C_{0}, N, n$, and $p$.

Now that we have stated Lemma 1, we go on to the proof of Theorem 2.

Proof of Theorem 2. Assume that $f \geq 0$. First we conclude that $M_{A^{p}} f \sim M_{A^{\prime p}} f$, where

$$
A_{\beta_{1}, \beta_{2}}^{\prime p}=\left\{\left(r e^{i \theta_{1}}, r e^{i \theta_{2}}\right): 1 / 2<r<1,\left|\beta_{j}-\theta_{j}\right| \leq(1-r)\left(\log \frac{1}{1-r}\right)^{p}, j=1,2\right\},
$$


because of Harnacks inequality, and the fact that $1-r_{1} \sim 1-r_{2}$.

Next we have that

$$
\begin{aligned}
& M_{A^{\prime p}} f\left(\beta_{1}, \beta_{2}\right) \\
& \sim \sup _{r, \theta_{1}, \theta_{2} \in A_{\beta_{1}, \beta_{2}}^{\prime p}} \frac{1}{\left(\log \frac{1}{1-r}\right)^{2}} \int_{\mathrm{T}^{2}} \frac{f\left(\varphi_{1}, \varphi_{2}\right) d \varphi_{1} d \varphi_{2}}{\left(1-r+\left|\theta_{1}-\varphi_{1}\right|\right)\left(1-r+\left|\theta_{2}-\varphi_{2}\right|\right)} \\
& =\sup _{r, \theta_{1}, \theta_{2} \in A_{\beta_{1}, \beta_{2}}^{\prime p}} \frac{1}{\left(\log \frac{1}{1-r}\right)^{2}}\left[\int_{D_{1}}+\int_{D_{2}}+\int_{D_{3}}+\int_{D_{4}}\right] \\
& \times \frac{f\left(\varphi_{1}, \varphi_{2}\right) d \varphi_{1} d \varphi_{2}}{\left(1-r+\left|\theta_{1}-\varphi_{1}\right|\right)\left(1-r+\left|\theta_{2}-\varphi_{2}\right|\right)},
\end{aligned}
$$

where

$$
\begin{aligned}
D_{1}= & \left\{\left(\varphi_{1}, \varphi_{2}\right):\left|\beta_{1}-\varphi_{1}\right| \leq 2(1-r)\left(\log \frac{1}{1-r}\right)^{p},\right. \\
& \left.\left|\beta_{2}-\varphi_{2}\right| \leq 2(1-r)\left(\log \frac{1}{1-r}\right)^{p}\right\}, \\
D_{2}= & \left\{\left(\varphi_{1}, \varphi_{2}\right):\left|\beta_{1}-\varphi_{1}\right| \leq 2(1-r)\left(\log \frac{1}{1-r}\right)^{p},\right. \\
& \left.\left|\beta_{2}-\varphi_{2}\right| \geq 2(1-r)\left(\log \frac{1}{1-r}\right)^{p}\right\}, \\
D_{3}= & \left\{\left(\varphi_{1}, \varphi_{2}\right):\left|\beta_{1}-\varphi_{1}\right| \geq 2(1-r)\left(\log \frac{1}{1-r}\right)^{p},\right. \\
& \left.\left|\beta_{2}-\varphi_{2}\right| \leq 2(1-r)\left(\log \frac{1}{1-r}\right)^{p}\right\}, \\
D_{4}= & \left\{\left(\varphi_{1}, \varphi_{2}\right):\left|\beta_{1}-\varphi_{1}\right| \geq 2(1-r)\left(\log \frac{1}{1-r}\right)^{p},\right. \\
& \left.\left|\beta_{2}-\varphi_{2}\right| \geq 2(1-r)\left(\log \frac{1}{1-r}\right)^{p}\right\} .
\end{aligned}
$$

Here, of course, $r, \theta_{1}, \theta_{2} \in A_{\beta_{1}, \beta_{2}}^{\prime p}$ means that $\left(r e^{i \theta_{1}}, r e^{i \theta_{2}}\right) \in A_{\beta_{1}, \beta_{2}}^{p}$. 
Let

$$
I_{i}(f)=\frac{1}{\left(\log \frac{1}{1-r}\right)^{2}} \int_{D_{i}} \frac{f\left(\varphi_{1}, \varphi_{2}\right) d \varphi_{1} d \varphi_{2}}{\left(1-r+\left|\theta_{1}-\varphi_{1}\right|\right)\left(1-r+\left|\theta_{2}-\varphi_{2}\right|\right)}
$$

and let $T^{i} f$ be the corresponding maximal function, $i=1, \ldots, 4$.

We now want to prove that each operator $T^{i}$ is of weak type $(p, p)$. We begin with $T^{1}$.

Preestimating $T^{1}$ by making a suitable partition of $D_{1}$ and estimate the kernel with a fixed value on each piece (see [1] for details), we have that

$$
\begin{aligned}
& T^{1} f\left(\beta_{1}, \beta_{2}\right) \leq C \sup _{\substack{1 / 2<r<1 \\
\left|\tau_{i}\right| \leq C(1-r)\left(\log \frac{1}{1-r}\right)^{p}}} \sum_{l=0}^{N(p, r)} \sum_{k=0}^{N(p, r)} \frac{1}{\left(\log \frac{1}{1-r}\right)^{2}} \frac{2^{-k} 2^{-l}}{(1-r)^{2}} \\
& \times \chi_{\left|x+\tau_{1}\right| \leq 2^{k}(1-r)} \chi_{\left|y+\tau_{2}\right| \leq 2^{l}(1-r)} * f\left(\beta_{1}, \beta_{2}\right) .
\end{aligned}
$$

Here we replaced $\theta_{i}-\beta_{i}$ by $\tau_{i}$. Let

$$
B_{j}=\left\{r: 2^{-2^{j}} \leq(1-r) \leq 2^{-2^{j-1}}\right\}, j \in Z^{+} .
$$

We see that if $r \in B_{j}$ then $N(p, r) \leq j p$, because $N(p, r)$ is determined by $2^{N(p, r)} \sim\left(\log \frac{1}{1-r}\right)^{p}$.

We thus have

$$
T^{1} f\left(\beta_{1}, \beta_{2}\right) \leq \sup _{j} T_{j} f\left(\beta_{1}, \beta_{2}\right)
$$

with

$$
\begin{gathered}
T_{j} f(\beta)=\sup _{\substack{r \in B_{j} \\
\left|\tau_{i}\right| \leq(1-r) 2^{j p}}} \sum_{k=0}^{N(p, r)} \sum_{l=0}^{N(p, r)} \\
\frac{1}{\left(2^{j}\right)^{2}} \frac{2^{-k} 2^{-l}}{(1-r)^{2}} \chi_{\left|x+\tau_{2}\right| \leq 2^{k}(1-r)} \chi_{\left|y+\tau_{2}\right| \leq 2^{l}(1-r)} * f\left(\beta_{1}, \beta_{2}\right) .
\end{gathered}
$$

We define

$$
\begin{aligned}
& T_{j k l} f\left(\beta_{1}, \beta_{2}\right) \equiv \sup _{\substack{r \in B_{j} \\
\left|\tau_{i}\right| \leq(1-r) 2_{j} p}} \frac{1}{2^{k} 2^{l}(1-r)^{2}} \\
& \times \chi_{\left|x+\tau_{1}\right| \leq 2^{k}(1-r)} \chi_{\left|y+\tau_{2}\right| \leq 2^{l}(1-r)} * f\left(\beta_{1}, \beta_{2}\right) .
\end{aligned}
$$

A straightforward calculation gives the following inequality: 
A CONVERGENCE RESULT FOR SQUARE ROOTS OF THE POISSON ...

$$
T_{j k l} f \leq C \frac{2^{2 j p}}{2^{k} 2^{l}} M f .
$$

Here $M f$ is the usual Hardy-Littlewood maximal function. This implies that $T_{j k l}$ is of weak type $(1,1)$ with norm less than $C 2^{2 j p} / 2^{k} 2^{l}$. From the definition we have that $T_{j k l} f$ is bounded on $L^{\infty}$ with norm $\sim 1$.

Marcinkiewicz's interpolation theorem now gives us that

$$
\left\|T_{j k l}\right\|_{\rho} \leq C(p, \rho) \frac{2^{2 j p / \rho}}{2^{k / \rho} 2^{l / \rho}}
$$

for $1<\rho<\infty$.

Thus we have

$$
\begin{aligned}
\left\|T_{j}\right\|_{\rho} & \leq \sum_{k, l=0}^{N(p, j)} \frac{1}{\left(2^{j}\right)^{2}}\left\|T_{j k l}\right\|_{\rho} \leq C(p, \rho) \sum_{k, l=0}^{N(p, j)} \frac{1}{\left(2^{j}\right)^{2}} \frac{2^{2 j p / \rho}}{2^{k / \rho} 2^{l / \rho}} \\
& =C(p, \rho) \sum_{k=0}^{N(p, j)} \frac{2^{j\left(\frac{p}{\rho}-1\right)}}{2^{k / \rho}} \sum_{l=0}^{N(p, j)} \frac{2^{j\left(\frac{p}{\rho}-1\right)}}{2^{l / \rho}} \sim 2^{2 j\left(\frac{p}{\rho}-1\right)} C(p, \rho) \leq C(p, \rho),
\end{aligned}
$$

if $\rho \geq p$.

This implies that if $\rho \geq p$ then $T_{j}$ is of weak type $(\rho, \rho)$ uniformly in $j$, so condition (1) in Lemma 1 is satisfied.

Let $\gamma_{j}=2^{c} 2^{j p+1} 2^{-2^{j-1}}$ and let

$$
\begin{aligned}
& K_{r, \tau_{1}, \tau_{2}}(x, y) \\
& =\sum_{k=0}^{N(p, r)} \sum_{l=0}^{N(p, r)} \frac{1}{\left(\log \frac{1}{1-r}\right)^{2}} \frac{1}{2^{k}(1-r)} \frac{1}{2^{l}(1-r)} \chi_{\left|x+\tau_{1}\right| \leq 2^{k}(1-r)} \chi_{\left|y+\tau_{2}\right| \leq 2^{l}(1-r)},
\end{aligned}
$$

that is $K_{r, \tau_{1}, \tau_{2}}(x, y)$ are the kernels of the operators in the definition of $T_{j}$.

It is easy to see that $\left\{\gamma_{j}^{\prime}\right\}=\left\{\left(\gamma_{j}, \gamma_{j}\right)\right\}$ satisfies the conditions of Lemma 1 after a suitable modification for small $j$, using that

$$
N(p, r) \leq p j \quad \text { if } \quad r \in B_{j} .
$$

Let $K^{*}$ be as in Lemma 1 . Let $r \in B_{j}$ and set $N=2$. Then we have 


$$
\begin{aligned}
& K_{r, \tau_{1}, \tau_{2}}^{*}(x, y)=\sup \left\{\sum_{k, l=0}^{N(\varphi, r)} \frac{1}{\left(\log \frac{1}{1-r}\right)^{2}} \frac{1}{2^{k}(1-r)} \frac{1}{2^{l}(1-r)}\right. \\
& \left.\times \chi_{\left|x+z_{1}+\tau_{1}\right| \leq 2^{k}(1-r)} \chi_{\left|y+z_{2}+\tau_{2}\right| \leq 2^{l}(1-r)}:\left|z_{i}\right| \leq \gamma_{j+2}, i=1,2\right\}
\end{aligned}
$$

and

$$
\begin{aligned}
& \int K_{r, \tau_{1}, \tau_{2}}^{*}(x, y) d x d y \\
& \leq \sum_{k, l=0}^{N(p, r)} \frac{1}{\left(\log \frac{1}{1-r}\right)^{2}} \int \frac{1}{2^{k}(1-r) 2^{l}(1-r)} \chi_{\left|x+\tau_{1}\right| \leq 2^{k}(1-r)+\gamma_{j+2}} \\
& \times \chi_{\left|y+\tau_{2}\right| \leq 2^{l}(1-r)+\gamma_{j+2}} d x d y \leq C(p, r),
\end{aligned}
$$

where $C(p, r)$ is bounded with respect to $r$ because $\gamma_{j+2} \leq C 2^{j p} 2^{-2^{j}}(1-r) \leq$ $C \min \left\{2^{k}(1-r), 2^{l}(1-r)\right\}$.

Thus, condition (2) of Lemma 1 is satisfied. The rest of the conditions in Lemma 1 are trivially satisfied, so the lemma gives that $T^{1}$ is at least of weak type $(p, p)$.

We next prove that $T^{2}$ is of weak type $(p, p)$ :

In the same way as before, we get that

$$
\begin{aligned}
& T^{2} f\left(\beta_{1}, \beta_{2}\right) \\
& =\sup _{r, \theta_{1}, \theta_{2} \in A_{\beta_{1}, \beta_{2}}^{\prime p}} \frac{1}{\left(\log \frac{1}{1-r}\right)^{2}} \int_{D_{2}} \frac{f\left(\varphi_{1}, \varphi_{2}\right) d \varphi_{1} d \varphi_{2}}{\left(1-r+\left|\theta_{1}-\varphi_{1}\right|\right)\left(1-r+\left|\theta_{2}-\varphi_{2}\right|\right)} \\
& \sim \sup \frac{1}{\left(\log \frac{1}{1-r}\right)^{2}} \int_{D_{2}} \frac{f\left(\varphi_{1}, \varphi_{2}\right) d \varphi_{1} d \varphi_{2}}{\left(1-r+\left|\theta_{1}-\varphi_{1}\right|\right)\left(1-r+\left|\beta_{2}-\varphi_{2}\right|\right)} \\
& \leq \sup _{\substack{1 / 2<r<1 \\
\left|\beta_{1}-\theta_{1}\right| \leq(1-r)\left(\log \frac{1}{1-r} p^{p}\right.}} \sum_{k=0}^{N(p, r)} \frac{1}{2^{k}(1-r)\left(\log \frac{1}{1-r}\right)^{2}} \int_{D_{2}^{k}} \frac{f\left(\varphi_{1}, \varphi_{2}\right) d \varphi_{1} d \varphi_{2}}{\left(1-r+\left|\beta_{2}-\varphi_{2}\right|\right)}
\end{aligned}
$$

where

$$
D_{2}^{k}=D_{2} \cap\left\{2^{k-1}(1-r) \leq\left|\theta_{1}-\varphi_{1}\right| \leq 2^{k}(1-r)\right\}
$$


We now observe that this can be written as

$$
\begin{aligned}
& \sup _{r, \tau} \sum_{k=0}^{N(p, r)} \frac{1}{2^{k}(1-r)\left(\log \frac{1}{1-r}\right)^{2}} \cdot \frac{1}{1-r+|y|} \chi_{|y|>2(1-r)\left(\log \frac{1}{1-r}\right)^{p}} \\
& \times \chi_{|x| \leq 2(1-r)\left(\log \frac{1}{1-r}\right)^{p}} \chi_{2^{k-1}(1-r) \leq|x+\tau| \leq 2^{k}(1-r)} * f\left(\beta_{1}, \beta_{2}\right),
\end{aligned}
$$

where $\tau=\theta_{1}-\beta_{1}, x$ corresponds to $\varphi_{1}$ and $y$ corresponds to $\varphi_{2}$.

We make the same partition of the variable $r$ into sets $B_{j}$ as before, to obtain

(5)

$$
\begin{aligned}
& T^{2} f\left(\beta_{1}, \beta_{2}\right) \leq C \sup _{j} \sup _{\substack{r \in B_{j} \\
|\tau| \leq(1-r) 2^{j p}}} \sum_{k=0}^{N(p, r)} \frac{1}{2^{k} 2^{2 j}(1-r)} \cdot \frac{1}{1-r+|y|} \\
& \times \chi_{|x+\tau| \leq 2^{k}(1-r)} \chi_{|y|>2(1-r)\left(\log \frac{1}{1-r}\right)^{p}} * f\left(\beta_{1}, \beta_{2}\right) \\
& \leq C \sum_{k=0}^{\infty} \frac{1}{\left(2^{1 / p}\right)^{k}} \sup _{j \geq k / p} \sup _{\substack{r \in B_{j} \\
|\tau| \leq(1-r) 2^{j p}}} \frac{2^{k / p}}{2^{k} 2^{2 j}(1-r)} \cdot \frac{1}{1-r+|y|} \chi_{|x+\tau| \leq 2^{k}(1-r)} \\
& \times \chi_{|y|>2(1-r)\left(\log _{1-r}\right)^{1}} * f\left(\beta_{1}, \beta_{2}\right),
\end{aligned}
$$

because $N(p, r) \leq j p$.

Now let $e_{l}=e^{-2^{l}}$ so that $|y|>2 e_{j} 2^{j p}$ if $r \in B_{j}$. Make the partition

$$
2 e_{j-\nu+1} 2^{j p} \leq|y| \leq 2 e_{j-\nu} 2^{j p}, \quad \nu=1,2, \ldots, j
$$

and estimate (5) with

$$
\begin{aligned}
& T^{2} f\left(\beta_{1}, \beta_{2}\right) \\
& \leq C \sum_{k=0}^{\infty} \frac{1}{\left(2^{1 / p}\right)^{k}} \sup _{j \geq k / p} \sup _{\substack{r \in B_{j} \\
|\tau| \leq(1-r) 2^{j p}}} \sum_{\nu=1}^{j} 2^{-\nu} \frac{2^{k / p}}{2^{k} 2^{2 j}(1-r)} \\
& \cdot \frac{2^{\nu}}{|y|+2 e_{j-\nu+1} 2^{j p}} \chi_{|x+\tau| \leq 2^{k}(1-r)} \chi_{2 e_{j-\nu+1} 2^{j p} \leq|y| \leq 2 e_{j-\nu} 2^{j p} * f\left(\beta_{1}, \beta_{2}\right)} \\
& \leq C \sum_{k=0}^{\infty} \frac{1}{\left(2^{1 / p}\right)^{k}} \sum_{\nu=1}^{\infty} 2^{-\nu} \sup _{\substack{j \geq \nu \\
j \geq k / p}} \sup _{\substack{|\tau| \leq B_{j} \\
\nu^{\prime}(1-r) j^{j p}}} \frac{2^{k / p}}{2^{j} 2^{k}(1-r)} \\
& \cdot \frac{2^{\nu-j}}{|y|+2 e_{j-\nu+1} 2^{j p}} \chi_{|x+\tau| \leq 2^{k}(1-r)} \chi_{|y| \leq 2 e_{j-\nu} 2^{j p}} * f\left(\beta_{1}, \beta_{2}\right)
\end{aligned}
$$




$$
\equiv C \sum_{k=0}^{\infty} \frac{1}{\left(2^{1 / p}\right)^{k}} \sum_{\nu=1}^{\infty} 2^{-\nu} \sup _{\substack{j \geq \nu \\ j \geq k / p}} 2^{k / p} 2^{-j} T_{j k \nu} f\left(\beta_{1}, \beta_{2}\right) .
$$

We are now going to use Lemma 1 to prove that $\sup _{j \geq \max (\nu, k / p)} 2^{k / p-j} T_{j k \nu} f$ is of weak type $(p, p)$. We first need to show that $T_{j k \nu}$ is of weak type $(p, p)$.

A straight estimate gives

$$
T_{j k \nu} f \leq \sup _{\substack{r \in B_{j} \\|\tau| \leq(1-r) 2^{j p}}} \frac{2^{j p}}{2^{k}} \frac{1}{2^{j p}(1-r)} \cdot \frac{2^{-j+\nu}}{|y|+2 e_{j-\nu+1} 2^{j p}} \chi_{|x| \leq 2 \cdot 2^{j p}(1-r)} \chi_{|y| \leq 2 e_{j-\nu} 2^{j p}} * f .
$$

Because $\frac{2^{-j+\nu}}{|y|+2 e_{j-\nu+1}{ }^{j p}} \chi_{|x| \leq 2 \cdot 2^{j p}(1-r)}$ is an $L^{1}$-funciton with norm independent of $j, k$ and $\nu$, Youngs inequality gives that the convolution in the $y$-variable in this estimate of $T_{j k \nu} f$, is a strong type $(1,1)$ operator with norm $\sim C$, where $C$ is independent of $j, k$ and $\nu$.

Because the convolution in the $x$-variable in essence gives a Hardy-Littlewood maximal operator, we have that $T_{j k \nu} f \leq C 2^{j p} / 2^{k} M^{\prime} f$ where $M^{\prime} f$ is a weak type $(1,1)$ operator with norm $\sim 1$. Going back to the definition of $T_{j k \nu}$, we easily have that $T_{j k \nu}$ is bounded on $L^{\infty}(\mathrm{T})$, uniformly in $\nu$, so Marcinkiewicz's interpolation theorem gives that $T_{j k \nu}$ is bounded on $L^{\rho}(\mathrm{T}), 1<\rho<\infty$, with $\left\|T_{j k \nu}\right\|_{\rho} \leq C(p, \rho)\left(\frac{2^{j p}}{2^{k}}\right)^{1 / \rho}$, uniformly in $\nu$.

Thus,

$$
\left\|2^{k / p-j} T_{j k \nu}\right\|_{\rho} \leq C(p, \rho) 2^{j p / \rho-j} 2^{k / p-k / \rho},
$$

so that $2^{k / p-j} T_{j k \nu}$ is bounded on $L^{\rho}(\mathrm{T})$ uniformly in $j$, if $\rho=p$.

It is easy to see that for large values of $j$

$$
\gamma_{j}^{\prime}=\left(2^{j p+1} e_{j-1}, 2^{j p+1} e_{j-\nu}\right)
$$

satisfies the conditions on the sequence $\left\{\gamma_{j}\right\}$ in Lemma 1 . If we modify $\gamma_{j}^{\prime}$ for small values of $\gamma$, we will get a satisfactory sequence $\left\{\gamma_{j}\right\}=\left\{\left(\gamma_{j, 1}, \gamma_{j, 2}\right)\right\}$.

We will next prove condition (2) of Lemma 1 . Because $j \geq k / p$ we can neglect the factor $2^{k / p-j}$ and will consider the kernels in the definition of $T_{j k \nu}$. Let $K_{r, \tau}^{j k \nu}$ be the kernel of the operator $T_{j k \nu}$, and let

$$
K_{r, \tau}^{j k \nu^{*}}(x, y)=\sup \left\{K_{r, \tau}^{j k \nu}\left(x+\varphi_{1}, y+\varphi_{2}\right) ;\left|\varphi_{1}\right| \leq \gamma_{j+N, 1},\left|\varphi_{2}\right| \leq \gamma_{j+N, 2}\right\},
$$

if $r \in B_{j}$. Here we let $N=2$.

We now estimate $K_{r, \tau}^{j k \nu}$ in the same way as we did when we proved that $T_{j k \nu}$ is of weak type $(1,1)$.

From this estimate we get that 


$$
\begin{aligned}
K_{r, \tau}^{j k \nu^{*}} & \leq \sup _{\varphi}\left\{\frac{1}{2^{k}(1-r)} \cdot \frac{2^{\nu-j}}{\left|y+\varphi_{2}\right|+2 e_{j-\nu+1} 2^{j p}} \chi_{\left|x+\varphi_{1}+\tau\right| \leq 2^{k+1}(1-r)}\right. \\
& \left.\times \chi_{\left|y+\varphi_{2}\right| \leq 2^{j p+1} e_{j-\nu}} ;\left|\varphi_{i}\right| \leq \gamma_{j+2, i} i=1,2\right\} \\
& \leq \sup _{\varphi}\left\{\frac{1}{2^{k}(1-r)} \cdot \frac{2^{\nu-j}}{\left|y+\varphi_{2}\right|+2 e_{j-\nu+1} 2^{j p}} \chi_{\left.|x+\tau| \leq 2^{k+1}+2^{j p+2 p+1} 2^{-2 j}\right)(1-r)}\right. \\
& \left.\times \chi_{\left|y+\varphi_{2}\right| \leq 2^{j p+1} e_{j-\nu}} ;\left|\varphi_{2}\right| \leq \gamma_{j+2,2}\right\} \\
& \leq \frac{C}{2^{k}(1-r)} \chi_{|x+\tau| \leq\left(2^{k+1}+2^{j p+2 p+1} 2^{-2 j}\right)(1-r)} \frac{2^{\nu-j}}{|y|+2 e_{j-\nu+1} 2^{j p}} \\
& \times \chi_{|y| \leq 2^{j p+1} e_{j-\nu}+2^{j p+p+1} e_{j-\nu+1}} .
\end{aligned}
$$

This implies

$$
\int K_{r, \tau}^{j k \nu^{*}}(x, y) d x d y \leq C
$$

for all $r$. Thus we have proved condition (2) in Lemma 1.

It is easy to see that the remaining conditions in the lemma are satisfied, and so we can apply the lemma to show that the operator $\sup _{j \geq \max (\nu, k / p)} 2^{k / p-j} T_{j k \nu}$ in (6) is of weak type $(p, p)$ uniformly in $\nu$ and $k$. Because of the coefficients in the summation in $k$ and $\nu$ in (6), we can estimate $T^{2}$ with a weak type $(p, p)$ operator. Thus $T^{2}$ itself is a weak type $(p, p)$ operator.

The proof for $T^{3}$ is identical to the proof of $T^{2}$, because of symmetry, so the only thing left is to prove that $T^{4}$ is of weak type $(p, p)$.

We have that

$$
\begin{aligned}
& T^{4} f\left(\beta_{1}, \beta_{2}\right) \\
& \leq \sup _{r, \theta_{1}, \theta_{2} \in A_{\beta_{1}, \beta_{2}}^{\prime p}} \frac{C}{\left(\log \frac{1}{1-r}\right)^{2}} \int_{D_{4}} \frac{f\left(\varphi_{1}, \varphi_{2}\right) d \varphi_{1} d \varphi_{2}}{\left(1-r+\left|\beta_{1}-\varphi_{1}\right|\right)\left(1-r+\left|\beta_{2}-\varphi_{2}\right|\right)} \\
& \leq C \sup _{1 / 2 \leq r<1} \sum_{k=1}^{M(p, r)} \frac{1}{\left(\log \frac{1}{1-r}\right)^{2}} \int_{D_{4}^{k}} \frac{f\left(\varphi_{1}, \varphi_{2}\right) d \varphi_{1} d \varphi_{2}}{\left(1-r+\left|\beta_{1}-\varphi_{1}\right|\right)\left(1-r+\left|\beta_{2}-\varphi_{2}\right|\right)} \\
& \sim C \sup _{1 / 2 \leq r<1} \sum_{k=1}^{M(p, r)} \frac{1}{\left(\log \frac{1}{1-r}\right)^{2}} \int_{D_{4}^{k}} \frac{f\left(\varphi_{1}, \varphi_{2}\right) d \varphi_{1} d \varphi_{2}}{2^{k}(1-r)\left(1-r+\left|\beta_{2}-\varphi_{2}\right|\right)}
\end{aligned}
$$


where $M(p, r) \sim \log \frac{1}{1-r}$ and $D_{4}^{k}=D_{4} \cap\left\{2^{k-1}(1-r) \leq\left|\beta_{1}-\varphi_{1}\right| \leq 2^{k}(1-r)\right\}$. We rewrite the supremum over $1 / 2<r<1$ as suprema over $j$ and $B_{j}$ and get the estimate

$$
T^{4} f \leq C \sup _{j} \sum_{k=1}^{M(p, j)} \sup _{r \in B_{j}} \frac{1}{2^{2 j}} \frac{1}{2^{k}(1-r)} \int_{D_{4} \cap\left\{\left|\varphi_{1}-\beta_{1}\right| \leq 2^{k}(1-r)\right\}} \frac{f\left(\varphi_{1}, \varphi_{2}\right) d \varphi_{1} d \varphi_{2}}{1-r+\left|\beta_{2}-\varphi_{2}\right|} .
$$

The operators in this majorant of $T^{4} f$ are of the same kind as those we used to estimate $T^{2} f$, and so $T^{4}$ is of weak type $(p, p)$.

Thus $M_{A^{p}}$ is of weak type $(p, p)$.

\section{REFERENCES}

1. J.-O. Rönning, Convergence results for the square root of the Poisson kernel, Math. Scand. 81 (1997), 219-235.

2. P. Sjögren, Une remarque sur la convergence des functions propres, du Laplacien à Valeur Propre Critique, Lecture Notes in Math. 1096 (1984), 544-548.

3. P. Sjögren, Convergence for the Square Root of the Poisson Kernel, Pacific J. Math. 131 (1988), 361-391.

INSTITUTIONEN FÖR NATURVETENSKAP HÖGSKOLAN I SKÖVDE BOX 408

54128 SKÖVDE

SWEDEN

Email:jan-olav@inv.his.se 